\title{
ALEXANDROFF ALGEBRAS AND COMPLETE REGULARITY
}

\author{
GEORGE REYNOLDS
}

\begin{abstract}
We characterize lattice theoretically the topological notion of complete regularity and study the implications of this characterization in the setting of local lattices ( $\equiv$ complete distributive lattices).
\end{abstract}

In this paper, we will give a lattice theoretic characterization of complete regularity. Normality, compactness and paracompactness among others, have been considered from the point of view of lattice theory, and since they make no reference to the points of the space they can be translated almost verbatim into the language of the theory of lattices. Complete regularity is awkward on two counts: not only does it mention the points of the space explicitly, it involves directly the real numbers. However with a maneuver inspired by the work of A. D. Alexandroff we will derive a characterization which makes no reference to the points of the space or the real numbers. An analogy can be made with paracompactness-this notion is defined in a lattice theoretic manner and has a well-known characterization involving real valued functions. Frink [3] has a characterization of complete regularity which avoids mention of the real numbers but involves the points of the space directly in the characterization. Moreover his proof appeals to the existence of ultrafilters by way of a Wallman compactification. The proof of our Theorem 1.4 appeals only to a variant of Urysohn's lemma which was proved in [13].

We draw the attention of the reader to [12] which characterizes complete regularity from another point of view. In Theorem 1.6 we will show that this result can be extended to any local lattice ( $\equiv$ complete distributive lattice) which is completely regular. In $\$ 2$ we show that the familiar construction of the Baire sets can be extended to this lattice theoretic setting also.

\section{Alexandroff algebras.}

1.1 Definition (see [8]). A local lattice $L$ is a complete lattice which satisfies the identity $x \wedge\left(\bigvee y_{\alpha}\right)=\bigvee\left(x \wedge y_{\alpha}\right)$ for any family $\left\{y_{\alpha}\right\} \subseteq L$. A homomorphism of local lattices is a function which preserves finite meets and arbitrary joins.

Observe that if $X$ and $Y$ are topological spaces then the open subsets of $X$ and $Y$ form local lattices, $\Theta(X)$ and $\Theta(Y)$, respectively, and the continuous functions from $X$ to $Y$ are in bijective correspondence with the homomorphisms from $\theta(Y)$ to $\theta(X)$ provided $X$ and $Y$ are sober (see [9]).

Received by the editors August 28, 1978 and, in revised form, December 1, 1978.

AMS (MOS) subject classifications (1970). Primary 54A05; Secondary 06A35, 18F20. 
1.2 Definition (see [1], [6], [13]). A lattice $L$ is a $\sigma$-lattice if $L$ has countable joins and satisfies the identity $x \wedge\left(\vee y_{\alpha}\right)=\bigvee\left(x \wedge y_{\alpha}\right)$ for all countable families $\left\{y_{\alpha}\right\} \subseteq L$. An Alexandroff algebra is a $\sigma$-lattice $A$ with 0 and 1 which satisfies the following two conditions:

(1) (normality) For all $a$ and $b$ in $A, a \bigvee b=1$ implies there exists $u$ and $w$ in $A$ with $u \wedge w=0, a \vee u=1$ and $b \vee w=1$.

(2) (implicit complements) To each $a$ in $A$, there is a sequence $\left(a_{i}, b_{i}\right)$ $(i \in \omega)$ in $A \times A$ with $b_{i} \vee a=1, b_{i} \wedge a_{i}=0$ and $\bigvee a_{i}=a$.

A homomorphism in Alexandroff algebras is a function preserving 0,1 finite meets and countable joins. $\mathbb{Q}$ will denote the category of Alexandroff algebras.

1.3 Definition. A local lattice $L$ is completely regular if $L$ contains an Alexandroff algebra $A$ as a $\sigma$-sublattice which generates $L$ by unrestricted joins. (We only note that by $\sigma$-sublattice, we mean that the inclusion $A \subseteq L$ preserves not only countable joins and finite meets, but 0 and 1 as well.)

Recall from [13] that every Alexandroff algebra is contained as a $\sigma$-sublattice of a local lattice $L$ making $L$ completely regular. We can now state the main result.

1.4 TheOREM. Let $X$ be a topological space with $\theta(X)$ the lattice of open sets. Then $X$ is completely regular as a space if and only if $\Theta(X)$ is completely regular as a local lattice.

First observe that if $X$ is completely regular as a space then the family $A=\operatorname{coz}(X)=\left\{f^{-1}(R-\{0\}) \mid f \in C(X)\right\}$ is an Alexandroff algebra (see [6]). Hence it suffices to show that if $\mathcal{O}(X)$ is completely regular as a local lattice, then $X$ is completely regular. The key to this implication is a variant of Urysohn's lemma. The precise statement follows and is proved in [13]. Recall that for an Alexandroff algebra $A$, a real valued function on $A$ is a homomorphism $h: \mathcal{O}(R) \rightarrow A$.

1.5 ThEOREM. If $A$ is an Alexandroff algebra and $a \in A$, then there is a real valued function $h$ on $A$ with $h(R-\{0\})=a$.

We can now complete the proof of Theorem 1.4. Let $A$ be an Alexandroff algebra which makes $\theta(X)$ completely regular. We will show that for every $U \in \mathcal{O}(X)$ and $x \in U$ there is a continuous function $f: X \rightarrow R$ with $f(x) \neq 0$ and $\left.f\right|_{X-U} \equiv 0$. Since $A$ generates $\theta(X)$, there is a $W \in A$ with $x \in W \subseteq U$. By Theorem 1.5, there is an $h: \vartheta(R) \rightarrow A$ with $h(R-\{0\})=W$. To obtain the function $f$ we proceed as follows: if $t \in X$, then

$$
\mathscr{F}_{t}=\{U \in \mathcal{O}(R) \mid t \notin h(U)\}
$$

is a prime ideal of open sets in $R$, closed under countable union. Hence $\cup \mathscr{F}_{t}=R-\{f(t)\}$ for some unique point $f(t)$. This defines $f: X \rightarrow R$. If $U$ is open in $R, f(t) \in U$ if and only if $U \notin \mathscr{F}_{t}$. Thus,

$$
f^{-1}(U)=\{t \mid f(t) \in U\}=\left\{t \mid U \notin \mathscr{F}_{t}\right\}=\{t \mid t \in h(U)\}=h(U),
$$


so $f$ is continuous. We deduce that $W=h(R-\{0\})=f^{-1}(R-\{0\})$, so $f(x) \neq 0$ and $\left.f\right|_{X-U} \equiv 0$. This completes the proof.

Let us say that a $\sigma$-sublattice $A$ contained in a local lattice $L$ is dense if every element of $L$ is a join of elements of $A$, and denote by $\operatorname{coz}(L)$ the $\sigma$-sublattice of $L$ given by $\{f(R-\{0\}) \mid f: \theta(R) \rightarrow L\}$. The next result follows easily from Theorem 1.5.

1.6 COROLlaRY. A local lattice $L$ is completely regular if and only if $\operatorname{coz}(L)$ is dense in $L$.

We will now show that a theorem of Mulvey (see [12]) characterizing complete regularity in the case of "spatial" local lattices extends to our more general setting. The proof we give is just a variation of Mulvey's proof, which shows that any reference he makes to the points of the space can be avoided.

If $L$ is a local lattice, $\operatorname{Mod}_{L} R_{L}$ will denote the category of $R_{L}$ modules in the category $\operatorname{sh}(L)$ of sheaves on $L$ (see [9]), with $L$ being given the canonical topology. $R_{L}$ denotes the Dedekind real numbers in $\operatorname{sh}(L)$.

1.7 THEOREM. $R_{L}$ is a generator in $\operatorname{Mod}_{L} R_{L}$ if and only if $L$ is completely regular.

We will follow Mulvey's notation and write

$$
R_{L}^{u}(v)=\{h: \theta(R) \rightarrow L \wedge v \mid h(R-\{0\}) \subseteq v \wedge u\}
$$

$R_{L}^{u}$ is a submodule of $R_{L}$, and the assumption that $R_{L}$ is a generator implies that $\bigoplus_{B} R_{L}^{\text {coz }}(f) \stackrel{\varphi}{\rightarrow} R_{L}$ is an epimorphism where the direct sum is taken over the set

$$
B=\{\cos (f) \mid \cos (f)<u, f: \theta(R) \rightarrow L\} .
$$

Let $1_{u}$ be the identity in the ring $R_{L}^{u}(u)=R_{L}(u)$. The fact that $\varphi$ is an epimorphism implies that there is a cover $\left\{u_{\alpha}\right\}$ of $u$ and $t_{\alpha} \in \bigoplus R_{L}^{\operatorname{coz}(f)}\left(u_{\alpha}\right)$ with $\varphi\left(t_{\alpha}\right)=\left.1_{u}\right|_{u_{\alpha}}$. Therefore each $u_{\alpha}$ is contained in some $\operatorname{coz}(f)<u$, so $u=\bigvee u_{\alpha}<\bigvee_{B} \operatorname{coz}(f)$, hence $\bigvee_{B} \operatorname{coz}(f)=u$.

Conversely suppose that $L$ is completely regular. Let $\varphi: M \rightarrow N$ be a map of $R_{L}$ modules with the property that $\varphi_{1}: M(1) \rightarrow N(1)$ is a surjection. We are to show that for any $u \in L$ and $t \in N(u)$, there is a cover $\left\{u_{\alpha}\right\}$ of $u$ and $t_{\alpha} \in M\left(u_{\alpha}\right)$ with $\varphi\left(t_{\alpha}\right)=\left.t\right|_{u_{\alpha}}$. The open cover required is supplied by the family of cozero sets $\{\operatorname{coz}(f) \mid \operatorname{coz}(f)<u\}$ since $L$ is completely regular. Indeed, we may assume that each $f$ has the property that $\left.f\right|_{u} \cdot t \in N(u)$ can be extended to a global section: set $g_{n}=(|f| \vee 1 / n)-1 / n$, and note that $0 \in N(f(\{x|| x \mid<1 / n\}))$ and $\left.g_{n}\right|_{u} \cdot t \in N(u)$ agree on $f(\{x|| x \mid<1 / n\}) \wedge$ $u$. Then observe that $\bigvee_{n} \operatorname{coz}\left(g_{n}\right)=\operatorname{coz}(f)$.

Now if $g_{k}=2\left(\left(\left(k|f| \wedge \frac{3}{4}\right) \bigvee \frac{1}{4}\right)-\frac{1}{4}\right), k \in N,\left.g_{k}\right|_{u} \cdot t$ can be extended to a global section $h_{k}$ and for sufficiently large $k,\left.\left.g_{k}\right|_{v_{k}} \cdot t\right|_{v_{k}}=\left.t\right|_{v_{k}}$ where $v_{k}=$ $f(\{x|| x \mid>1 / k\})$. Thus if $s_{k} \in M(1)$ with $\varphi\left(s_{k}\right)=h_{k}$, then $\left.s_{k}\right|_{v_{k}} \in M\left(v_{k}\right)$ is the desired collection. 
1.8 Corollary. If $A$ is an Alexandroff algebra then $R_{A}$ is a generator in the category of $R_{A}$ modules in $\operatorname{sh}(A), A$ being given the Grothendieck topology of "countable covers" (see [13]).

This follows since $A$ is dense in the local lattice $L$ of subobjects of 1 in $\operatorname{sh}(A)$, so $L$ is completely regular.

2. Baire sets. An important construction in measure theory associated with any completely regular space is that of the Baire sets, the $\sigma$-algebra generated by the cozero sets. This is a construction which also has the appearance of depending on the points of the space, in particular the construction is carried out inside the power set of $X$. We will show that to every Alexandroff algebra $A$ there is a naturally associated $\sigma$-complete boolean algebra which has every right to be called the Baire sets generated by $A$. Precisely:

2.1 THEOREM. The category of $\sigma$-complete boolean algebras is a full reflective subcategory of the category of Alexandroff algebras, and the reflection functor applied to the cozero sets of a topological space $X$ yields exactly the Baire sets of $X$.

That the inclusion functor from $\sigma$-complete boolean algebras to Alexandroff algebras has a left adjoint is a standard (and very general) application of the general adjoint functor theorem, and we shall omit the details. For any Alexandroff algebra $A$, let $\mathscr{B}(A)$ be the $\sigma$-complete boolean algebra obtained by the above process. If $X$ is a topological space, let $\eta$ : $\operatorname{coz}(X) \rightarrow \mathscr{B}(\operatorname{coz}(X))$ be the front adjunction map, $i: \operatorname{coz}(X) \rightarrow \mathscr{B} a(X)$ the inclusion of $\operatorname{coz}(X)$ into the Baire sets of $X, j: \mathscr{B}(\operatorname{coz}(X)) \rightarrow \mathscr{B} a(X)$ the unique homomorphism of $\sigma$-complete boolean algebras insured by the adjointness. Then the image of $j$ is a $\sigma$-complete boolean algebra containing $\operatorname{coz}(X)$, so $j$ is onto. Let $\mathscr{B}_{0}=\eta[\operatorname{coz}(X)]$ and let $B_{\lambda}, \lambda<\omega_{1}$, be the usual "Borel" hierarchy obtained inductively from $\mathscr{B}_{0}$ by taking complements and countable unions. Note that

$$
\bigcup_{\lambda<\omega_{1}} \mathscr{B}_{\lambda}=\mathscr{B}(\operatorname{coz}(X))
$$

since as a $\sigma$-complete subalgebra of $\mathscr{B}(\operatorname{coz}(X)), \cup_{\lambda<\omega_{1}} \mathscr{B}_{\lambda}$ satisfies the same universal property that $\mathscr{B}(\operatorname{coz}(X))$ satisfies. Now observe that $\mathscr{B}_{0}$ has the following property:

(*) If $a$ or $a^{c}$ (the complement of $a$ ) is in $\mathscr{B}_{0}$, then $j(a)=0$ implies $a=0$.

We shall prove by induction that (*) is true when $\mathscr{B}_{0}$ is replaced by $\mathscr{B}_{\lambda}$ for all $\lambda<\omega_{1}$. This will clearly show that the kernel of $j$ is 0 , so $j$ is 1-to- 1 .

If $\mathscr{B}_{\alpha}$ has the property (*) for $\alpha<\lambda$ and $\lambda$ is a limit ordinal then (*) is obviously true of $\mathscr{B}_{\lambda}$. If $\lambda=\alpha+1$, and $b \in \mathscr{P}_{\lambda}$, then $b=\cup_{i \in \omega} a_{i}$ where $a_{i}$ or $a_{i}^{c} \in \mathscr{B}_{\alpha}$. Thus $j(b)=0$ implies $j\left(a_{i}\right)=0$ for all $i$, hence by the induction hypothesis, $a_{i}=0$ for all $i$, so $b=0$. This completes the proof.

Since the map from $A \rightarrow \mathscr{B}(A)$ is a monomorphism and preserves count- 
able joins, standard techniques from measure theory can be applied to obtain the last result whose proof we leave to the interested reader.

2.2 TheOREM. Let $A$ be an Alexandroff algebra. If $\mu: A \rightarrow[0,1]$ is a function which satisfies

(a) $\mu(a \bigvee b)=\mu(a)+\mu(b)$ if $a \wedge b=0$, and

(b) $\mu\left(\bigvee_{i} a_{i}\right)=\sup _{i} \mu\left(a_{i}\right)$ whenever $a_{i}<a_{i+1}$, then $\mu$ can be extended uniquely to a countably additive measure on $\Re(A)$.

\section{REFERENCES}

[1] A. D. Alexandroff, Additive set-functions in abstract spaces, Mat. Sb. 50 (1940), 307-348; 51 (1941), 563-628; 55 (1943), 169-238.

[2] R. Engelking, Outline of general topology, North-Holland, Amsterdam, 1968.

[3] O. Frink, Compactifications and semi-normal spaces, Amer. J. Math. 86 (1964), 602-607.

[4] L. Gillman and M. Jerison, Rings of continuous functions, Van Nostrand, Princeton, N. J., 1960.

[5] H. Gordon, Rings of functions determined by zero sets, Pacific J. Math. 36 (1971), 133-157.

[6] A. W. Hager, Real-valued functions on Alexandroff (zero-set) spaces, Comment Math. Univ. Carolinae 16 (1975), 755-769.

[7] E. Hewitt, Rings of real-valued continuous functions. I, Trans. Amer. Math. Soc. 64 (1948), 54-99.

[8] J. Isbell, Atomless parts of spaces, Math. Scand. 31 (1972), 5-32.

[9] P. Johnstone, Topos theory, Academic Press, New York, 1977.

[10] F. E. J. Linton, Functorial measure theory, (Proc. of the Conf. held at Univ. of California, Irvine), Thompson, Washington, D. C., 1967, pp. 36-49.

[11] S. Mac Lane, Categories for the working mathematician, Springer-Verlag, New York, 1971.

[12] C. Mulvey, A categorical characterization of compactness, J. London Math. Soc. (2) 17 (1978), 356-362.

[13] G. Reynolds, On the spectrum of a real representable ring, Proc. London Math. Soc. Sympos. of Applications of Sheaves (1977) (to appear).

Department of Mathematics, Union College, Schenectady, New York 12308 